\title{
The potential impacts of obesity on COVID-19
}

\author{
Author: Ahmed Abdalazim Dafallah Albashir ${ }^{\mathrm{A}}$
}

\begin{abstract}
Obese patients are at increased risk of exacerbations from viral respiratory infections. During the H1N1 pandemic, obesity was associated with an increased risk of influenzaassociated intensive care unit (ICU) admission and death, longer duration of mechanical ventilation, and longer duration of ICU and hospital length of stay compared with the nonobese. These observations have raised a concern about the correlation between obesity and the current COVID-19 pandemic. In this review, we have outlined the potential impacts of obesity on respiratory physiology and the function of both innate and adaptive immune responses. Also, it has been clearly illustrated that obese patients are potentially more vulnerable to COVID-19 and more contagious than lean patients. The comorbidities associated with obesity were found to be correlated with a severe clinical course of COVID-19 and increased mortality and high BMI has been shown to be correlated with hospitalisation, the need for mechanical ventilation and non-survival. The review also sheds light on the challenges that obese patients pose for healthcare providers inside and outside ICUs.
\end{abstract}

KEYWORDS: COVID-19, obesity, respiratory physiology, immunity, comorbidities

DOI: $10.7861 /$ clinmed.2020-0239

\section{Introduction}

Coronavirus disease 2019 (COVID-19) is a current pandemic outbreak caused by a novel coronavirus now called severe acute respiratory syndrome coronavirus 2 (SARS-CoV-2). The virus is an enveloped RNA virus found in humans and wildlife that was first identified in Wuhan City, Hubei Province, China. Epidemiological investigations revealed an initial connection with a seafood market that sold live animals, which was then closed for disinfection. ${ }^{2}$ However, the epidemic progressed, with the main mode of transmission being person-to-person spread. COVID-19 was first reported to the World Health Organization (WHO) on 31 December 2019. On 30 January 2020, the WHO declared the COVID-19 to be a world pandemic disease. ${ }^{3}$

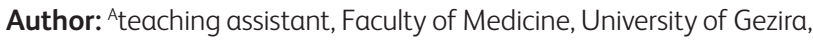
Sudan and resident, Wad Medani Teaching Hospital, Wad Madani City, Sudan
Older patients with comorbidities such as chronic pulmonary diseases, heart diseases, kidney diseases, diabetes and hypertension were vulnerable to a more severe course of the disease with higher mortality rates. According to a CDC report, the commonest underlying conditions among those hospitalised with COVID-19 were diabetes, chronic lung disease, and cardiovascular disease. ${ }^{4}$ Obesity is well known to be a risk factor for cardiovascular diseases and diabetes mellitus. Besides, many respiratory complications were associated with obesity, including increased demand for ventilation, increased work of breathing, respiratory muscle inefficiency, and diminished respiratory compliance. ${ }^{5}$ When compared to China, obesity is more prevalent in Italy, which might contribute to the different fatality rates between the two countries. ${ }^{6}$ Another point of note is that the United States, which currently has the highest mortality rates from COVID-19, has a high prevalence of obesity compared to China when obesity is defined by BMI.? Also, from the previous experience of the impacts of obesity on mortality during the H1N1 influenza epidemic, several challenges have been encountered when dealing with obese patients inside and outside the intensive care units (ICUs). All of these observations have raised concerns about the impacts that obesity could have on COVID-19.

\section{The impacts of obesity on respiratory physiology}

COVID-19 pneumonia has at least two distinct phenotypes, low $(\mathrm{L})$ phenotype and high $(\mathrm{H})$ phenotype. The $\mathrm{L}$ phenotype is characterised by low elastance (nearly normal/normal compliance which means that the amount of gas in the lung is almost normal), low ventilation-to-perfusion (VA/Q) ratio, low lung recruitability (very low amount of non-aerated tissue), and yet substantial hypoxemia. As the volume of gas is almost normal, hypoxemia is thought to be attributable to the loss of regulation of perfusion and loss of hypoxic vasoconstriction (pulmonary arterial vasoplegia). In this situation, the factor limiting pulmonary function will be perfusion. The normal response to hypoxemia is by increasing minute ventilation, primarily by increasing the tidal volume. The near-normal compliance explains why some COVID-19 patients have no dyspnea, as the patient inhales the volume that he expects.

On the other hand, the $\mathrm{H}$ phenotype is characterised by high elastance, low compliance, high right-to-left pulmonary shunts and high recruitability. In this case, the factor limiting pulmonary function will be ventilation. The type $\mathrm{H}$ pattern fully fits the criteria of severe ARDS (hypoxemia, bilateral infiltrates, decreased respiratory system compliance, increased lung weight and potential for recruitment).

In general, obesity is characterised by a decrease in total respiratory system compliance. This is partially due to a decrease 
in lung compliance, which could be related to the increased pulmonary blood volume seen in obese subjects. The primary reason, however, is the decrease in chest wall compliance that is due to accumulation of fat in and around the ribs and diaphragm and in the abdomen. The impacts of obesity on respiratory physiology also include increased airway resistance and work of breathing, respiratory muscle inefficiency, and ventilationperfusion inequality. It also associated with a symmetrical decrease in forced expiratory volume in one second (FEV-1) and forced vital capacity (FVC) in mild to moderate obesity. ${ }^{5}$ Severe obesity is associated with a greater decrease in FEV-1.

Obese subjects spend considerable energy to overcome the reduction in chest wall compliance (the elastic work). In addition, considerable energy must also be spent to overcome the air flow limitation and the airway resistance (the non-elastic work). The obese must also dedicate a disproportionately high percentage of total oxygen consumption $\left(\mathrm{VO}_{2}\right)$ to respiratory work, even during quiet breathing. This relative inefficiency indicates a decreased ventilatory reserve and a propensity to respiratory failure, in the setting of even mild systemic or pulmonary insults. Taken together, the already attenuated respiratory system of obese subjects can be severely compromised from any respiratory insults, meaning that these patients will struggle to recover if they have developed any severe illnesses that can deleteriously affect the respiratory function, like COVID-19.

To date, there are no published studies that mention the relation between obesity and the ventilator phenotypes of COVID-19. However, an increased prevalence of obesity has been found among patients with SARS-CoV-2 pneumonia with ventilatory phenotype $\mathrm{H}$ (ARDS-like). From the ventilatory point of view, these patients need high positive end-expiratory pressure (PEEP) and respond well to prone positioning and extracorporeal support. ${ }^{8}$

\section{The vulnerability of obese populations to contracting COVID-19}

Studies have shown that the SARS-CoV receptor ACE2 is used by the SARS-CoV-2 spike protein as a co-receptor for host cell entry. ${ }^{9}$ ACE2 is highly expressed in the heart and lungs. In adipose tissue, the expression of ACE2 is higher than in lung tissue, the main target tissue affected by COVID-19.10 Adipose tissue might also be a target site for COVID-19 infection. Obese subjects, who have an increased amount of adipose tissue, have an increased number of ACE2-expressing cells and therefore a larger amount of ACE2. However, there was no difference in adipocyte expression of ACE2 protein between obese individuals and those without obesity. ${ }^{11}$ These findings might contribute to the hypothesis that obese populations are at increased risk for COVID-19 infection.

After the initial engagement of the SARS-CoV-2 spike protein to the ACE2 receptor, there is downregulation of the membrane-bound ACE2. This downregulation of ACE2 activity in the lungs will lead to an unopposed accumulation of angiotensin II, the substrate for ACE2. Angiotensin II (ANG II) accumulation will lead to increased neutrophil accumulation, increased vascular permeability, and exacerbated pulmonary oedema, which will eventually lead to ARDS.

Obesity, both in humans and in experimental animals, is associated with an imbalance in the renin-angiotensin-aldosterone system (RAAS), resulting in an over-expression of the angiotensin II (ANG II) and angiotensin II receptor type 1 (AT1R) axis both at the level of adipose tissue and at systemic level. Moreover, the expression of ACE2 is considerably greater in visceral adipose tissue than in the subcutaneous adipose tissue. As long as adipose tissue can act as a reservoir for influenza A virus, HIV, human adenovirus Ad-36, and cytomegalovirus, SARS-CoV-2 can also use the adipose tissue as a reservoir to affect other parts of the body..$^{12}$ Upon that, obese subjects, especially those with extensive visceral adipose tissue, may develop an explosive systemic response of the ANG II and AT1R axis and could host and store an expansive viral load, which might lead to the development of a more severe form of the disease.

\section{Obesity and comorbidities}

Depending on the degree, duration, and distribution of the excess adipose tissue, obesity can progressively cause and/or exacerbate a variety of comorbidities, including hypertension, type 2 diabetes mellitus, dyslipidemia, cardiovascular diseases..$^{13}$ According to many studies, comorbidities including cardiovascular disease, hypertension, diabetes mellitus, and chronic lung diseases $4,14,15$ have been found to be associated a with severe clinical course of COVID-19 and increased mortality. Therefore, obesity can potentially contribute to increased morbidity and mortality among COVID-19 patients.

\section{The impacts of obesity on immunity}

Generally, obesity is characterised by a state of chronic and low-grade inflammation and obese patients are affected at various levels of their innate and adaptive immune responses. ${ }^{16}$ Expanded adipose tissue seen in obese adults is mainly due to adipocyte hypertrophy rather than adipocyte hyperplasia. ${ }^{17}$ Such hypertrophic lipid-engorged adipocytes are more likely to activate endoplasmic reticulum and mitochondrial stress responses along with mechanical stress-inducing shear on the extracellular environment. These factors together promote the activation of a chronic, pro-inflammatory state within the adipose tissue. ${ }^{18}$

Obese patients have chronically lowered concentration of adiponectin (an anti-inflammatory adipokine) and higher levels of leptin (a pro-inflammatory adipokine). ${ }^{19}$ There is an excess production of leptin and other pro-inflammatory cytokines such as TNF alpha, IL-6, MCP-1, and IL-1 3 in adipose tissue, which leads to pro-inflammatory excess energy milieu. The abundance of these pro-inflammatory mediators in adipose tissue leads to dysfunction of the innate immunity under obesity. ${ }^{20}$ Also, such an increase is associated with increased tissue inflammation and increased mortality upon infection in diet-induced obesity model (DIO model), ${ }^{21}$ which uses animals that have obesity caused by being fed high-fat or high-density diets. The chronic inflammatory state also blunts the activation of macrophages when an antigen is presented. Moreover, it reduces the pro-inflammatory cytokine production upon macrophage stimulation. ${ }^{22}$ This blunted activation of macrophages upon antigen presentation may explain the failure of vaccination in obese people.

Diabetes mellitus is reported as one of the commonest comorbidities associated with COVID-19 hospitalisations. ${ }^{4}$ The excess TNF alpha impairs the translocation of glucose transporter type 4 to the surface of skeletal muscle cells, leading to an increased mobilisation of free fatty acids (FFAs) from adipose into the circulation. ${ }^{23,24}$ Free fatty acids and triglycerides (TGs) are released into the bloodstream, which leads to the accumulation of lipid derivatives in skeletal muscle, liver, and pancreatic $\beta$-cells. This accumulation results in impaired tissue functioning and systemic insulin resistance. Another addressable factor for the insulin resistance is that the abundance of TNF alpha, leptin, and other pro-inflammatory mediators block the production of insulinsensitising adipokines such as adiponectin. ${ }^{25}$ 
Human studies revealed that the obese state is associated with either increased or decreased total lymphocytes in the peripheral blood. ${ }^{26-28}$ The CD8+ T cells are decreased and CD4+T cells are increased or decreased. ${ }^{28,29} \mathrm{~B}$ and $\mathrm{T}$ cell responses are also disrupted in obese patients, with reduced lymphocyte proliferative response. These disrupted responses result in increased sensitivity and delay in the resolution of viral infections. ${ }^{30}$ Obesity is also characterised by impaired memory CD8 $+T$ cell responses to influenza virus infections, resulting in increased mortality, elevated viral titers in lung and worsened lung pathology. ${ }^{31}$ Such adverse effects were accompanied by an obesity-induced inability to produce and maintain influenza-specific CD $8+$ memory T cells which are important for the effectiveness of the vaccine. ${ }^{31}$

Zhang et al hypothesized that leptin was a cofactor for the H1N1 pandemic in 2009..$^{32}$ The metabolic dysfunctions that are associated with obesity often lead to systemic leptin resistance. ${ }^{33}$ This resistance is associated with reduced bone marrow hematopoiesis and decreased T cell production and development in the thymus. ${ }^{34,35}$ Together,these disturbances result in diminished initiation of the immune responses, decreased memory $T$ cell reactions and decreased efficacy of vaccination. ${ }^{36}$ This impaired pro-inflammatory response has led to the severe lung lesions seen in patients of the H1N1 pandemic.

\section{Obese patients are potentially more contagious than lean people}

Studies have shown that obese patients are potentially more contagious than lean subjects in the setting of viral infections. Firstly, obesity increases the duration of viral shedding; symptomatic obese patients shed the virus $42 \%$ longer than people who do not have obesity. ${ }^{37}$ Secondly, the obesity state is characterised by a delayed capacity to produce interferons in human and animal studies. ${ }^{38,39}$ This delay in the production of the interferon enables further viral RNA replication to increase the chances of new, more virulent viral strains emerging. ${ }^{39}$ Thirdly, BMI was found to be positively associated with the level of infectious virus in the exhaled air. ${ }^{40}$

\section{Obesity in the clinical setting}

Previous experience of the impacts of obesity on H1N1 Influenza mortality shows that obese patients who become ill and required intensive care pose many challenges regarding their management. They are more challenging to intubate and to image. Due to the challenge of acquiring venous access, obesity has been shown to increase the risk of ICU acquired catheter and bloodstream infections. ${ }^{41}$ The obese are also more difficult to position and transport by the nursing staff and at higher BMI levels may need special beds and positioning/transport facilities that are not often available outside specialised bariatric surgical units. ${ }^{42}$

Another point of great concern is the fact that self-isolated obese people who prefer to avoid social interaction are already been stigmatised. And as social isolation may be misconstrued around their obesity, the healthcare providers need to guard against obesity stigma more than ever before.

\section{Early data}

The identification of obesity as an independent risk factor for hospitalisation and death in H1N1 influenza ${ }^{43}$ led to suspicion that it would have a significant impact on the current COVD-19 pandemic and concerns about the effects of obesity on COVID-19 have been further substantiated by preliminary data from many hospitals

One of the first published studies of BMI data was a descriptive study of a small group of 24 critically ill patients (64\% male) diagnosed with COVID-19 in the Seattle area, Washington (three patients were in normal category BMI, seven were overweight, 13 were obese and one had missing data). The study found that $85 \%$ of obese patients required mechanical ventilation and $62 \%$ of obese patients died. These proportions are higher than those in non-obesity patients, where $64 \%$ needed mechanical ventilation and $36 \%$ died. .4

In a study performed in the Third People's Hospital of Shenzhen, China from 11 January to 16 February 2020, 383 admitted patients with COVID-19 were included, and they were classified according to BMI as underweight (BMI lower than $18.5 \mathrm{~kg} / \mathrm{m}^{2}$ ), normal weight (18.5-23.9 kg/m²), overweight $\left(24.0-27.9 \mathrm{~kg} / \mathrm{m}^{2}\right)$, and obese $\left(\geq 28 \mathrm{~kg} / \mathrm{m}^{2}\right)$. ${ }^{45}$ The study concluded that overweight patients were associated with an $86 \%$ higher risk, and obesity with a $142 \%$ higher risk, of developing severe pneumonia compared with normal weight patients.

In another retrospective study of 112 patients with COVID-19 infection admitted to the western district of Union Hospital in Wuhan, the authors found that the highest BMI was seen more often in serious cases and non-survivors. ${ }^{46}$ Thromboembolic events were an important cause of death, and as long as obesity status is recognised as an important cause of thromboembolic events, obesity can be considered as an aggravating risk factor for death from COVID-19 infection. ${ }^{47}$

Using the ISARIC WHO Clinical Characterization Protocol, the features of 16,749 hospitalised patients with COVID-19 in the UK have been studied. This prospective observational cohort study has been performed in 166 UK hospitals during the period between 6 February and 18 April 2020. Chronic cardiac disease was the most common documented comorbidity (29\%). Next in order of frequency were uncomplicated diabetes (19\%), nonasthmatic chronic pulmonary disease (19\%) and asthma (14\%), whereas $47 \%$ were found to have no reported comorbidity. Of the hospitalised patients, $17 \%$ required critical care. Poor outcomes were associated with being elderly, male and obese. In addition to increased age, comorbidities including obesity were found to be associated with a higher probability of mortality. ${ }^{48}$ ISARIC, based on hospital survey data (2,212 deaths), revealed a hazard ratio (HR) of 1.37 for death linked to clinician-reported obesity among 16,749 hospitalised patients, after correction of age and sex $(95 \%$ confidence interval 1.16-1.63).

Recently, a large cohort study examined the factors associated with COVID-19-related hospital death in the linked electronic health records of 17 million adult NHS patients. It found that the risk of death increased with the degree of obesity: fully adjusted HR was 1.27 for BMI $30-34.9 \mathrm{~kg} / \mathrm{m}^{2}$, increasing to 2.27 for BMI $\geq 40 \mathrm{~kg} / \mathrm{m}^{2} .49$ According to the Intensive Care National Audit and Research Centre (ICNARC) report on 8,699 patients with COVID-19 being treated in ICUs in England (released 15 May 2020), BMI distribution revealed that $35.2 \%$ of the patients were overweight (BMI 25-30 kg/m²), 31.0\% were obese (BMI $\left.30-40 \mathrm{~kg} / \mathrm{m}^{2}\right)$, and $7.6 \%$ classified as having morbid obesity (BMI $\left.>40 \mathrm{~kg} / \mathrm{m}^{2}\right)$.

Newly released statistics from New York indicated that for those under the age of 60 , obesity was twice more likely to result in COVID-19 hospitalisation. The study, which included 4,103 patients with COVID-19 at an academic health system in New York City, showed that BMI $>40 \mathrm{~kg} / \mathrm{m}^{2}$ was the second strongest independent predictor of hospitalisation after old age..$^{50}$ 
Finally, in a small study from a university hospital in Lille, France, which enrolled 124 patients with COVID-19, the need for invasive mechanical ventilation was associated with a BMI of $\geq 35$, regardless of other comorbidities.

\section{Conclusion}

In conclusion, obesity is a well-recognised cause of respiratory function compromise which might make these group of patients at risk of a more severe clinical course if they contract COVID-19. We have also concluded that obese subjects are potentially more vulnerable to become infected with COVID-19, and are more contagious when they do so with a prolonged period of viral shedding. Obesity has been found to be a strong independent risk factor for hospitalisation in COVID-19, and to cause or exacerbate a variety of comorbidities which have been proven to be associated with increased morbidity and mortality among COVID-19 patients. Moreover, obese individuals have defective responses of both innate and adaptive immunity, with decreased response to vaccinations. And finally, obese patients are likely to strain the health system as they pose many challenges to healthcare provision inside and outside the ICUs. Results from many recently published studies have led to the conclusion that obesity is associated with poor outcomes in COVID-19, with a higher probability of mortality. Measurement of anthropometric characteristics for obesity is vital in both the primary care setting and in the hospital setting for a better estimation of the risk of complications in COVID-19 patients.

\section{Key points}

Special attention should be paid to obesity when treating COVID-19 patients.

For better estimation of the risk of complications in COVID-19 patients, it is very important to measure anthropometric parameters.

Obesity is a strong independent risk factor for hospitalisation in COVID-19.

Obesity increases the need for critical care and invasive mechanical ventilation due to COVID-19.

Obese patients are vulnerable to an adverse clinical course of COVID-19 and have a more contagious state than the lean patients.

Obese populations have a decreased response to vaccinations.

Obesity is associated with a variety of comorbid conditions that have been shown to be associated with increased morbidity and mortality from COVID-19.

Obese patients are more challenging to intubate, to diagnose via imaging, and for nursing staff to position and transport, and may require special beds and positioning/transport facilities.

From the ventilatory point of view, obese patients need high positive end-expiratory pressure (PEEP) and respond well to prone positioning and extracorporeal support.

\section{References}

1 Centers for Disease Control and Prevention. 2019 Novel Coronavirus, Wuhan, China. CDC, 2020. Available from www.cdc. gov/coronavirus/2019-ncov/about/index.html [Accessed 27 ]anuary 2020].

2 World Health Organization. Novel coronavirus (2019-nCoV) situation report - 2. WHO, 2020. Available from www.who.int/docs/defaultsource/coronaviruse/situation-reports/20200122-sitrep-2-2019-ncov. pdf [Accessed 23 January 2020].

3 Wee S-L, McNeil GD, Hernández JC. W.H.O. declares global emergency as Wuhan coronavirus spreads. New York Times, 30 January 2020.

4 Centers for Disease Control and Prevention COVID-19 Response Team. Preliminary estimates of the prevalence of selected underlying health conditions among patients with coronavirus disease 2019 - United States, February 12 - March 28, 2020. MMWR 2020;69;382-6.

5 Parameswaran K, Todd DC, Soth M. Altered respiratory physiology in obesity. Can Respir ] 2006;13:203-10.

6 Onder G, Rezza G, Brusaferro S. Case-fatality rate and characteristics of patients dying in relation to COVID-19 in Italy. JAMA 2020, in press (doi: 10.1001/jama.2020.4683).

$7 \mathrm{Hu}$ C, Jia W. Diabetes in China: epidemiology and genetic risk factors and their clinical utility in personalized medication. Diabetes 2018;67:3-11.

8 Costa H, Jacob M, Pereira R, Calças R. COVID-19 ventilatory phenotypes and obesity: is there a relationship? Obesity (Silver Spring) 2020, in press (doi: 10.1002/oby.22877).

9 Hoffmann M, Kleine-Weber H, Schroeder S et al. SARS-CoV-2 cell entry depends on ACE2 and TMPRSS2 and is blocked by a clinically proven protease inhibitor. Cell 2020;181:271-80.

10 Jia X, Yin C, Lu S et al. Two things about COVID-19 might need attention. Preprints 2020, 2020020315.

11 Pinheiro TA, Barcala-Jorge AS, Andrade JMO et al. Obesity and malnutrition similarly alter the renin-angiotensin system and inflammation in mice and human adipose. J Nutr Biochem 2017;48:74-82.

12 Bourgeois C, Gorwood J, Barrail-Tran A et al. Specific biological features of adipose tissue, and their impact on HIV persistence. Frontiers Microbiol 2019;10:2837.

13 Kyrou I, Randeva HS, Tsigos C et al. Clinical problems caused by obesity. In: Feingold KR, Anawalt B, Boyce A et al (eds), Endotext. South Dartmouth (MA): MDText.com, Inc; 2000-2020.

14 Zhou F, Yu T, Du R et al. Clinical course and risk factors for mortality of adult inpatients with COVID-19 in Wuhan, China: a retrospective cohort study. Lancet 2020;395:1054-62.

15 Wu Z, McGoogan JM. Characteristics of and important lessons from the coronavirus disease 2019 (COVID-19) outbreak in China: summary of a report of 72314 cases from the Chinese Center for Disease Control and Prevention. JAMA 2020, in press (doi: 10.1001/jama.2020.2648).

16 Andersen C], Murphy KE, Fernandez ML. Impact of obesity and metabolic syndrome on immunity. Adv Nutr 2016;7:66-75.

17 Naaz A, Holsberger DR, Iwamoto GA et al. Loss of cyclin-dependent kinase inhibitors produces adipocyte hyperplasia and obesity. FASEB J 2004;18:1925-7.

18 Deng J, Liu S, Zou L et al. Lipolysis response to endoplasmic reticulum stress in adipose cells. J Biol Chem 2012;287:6240-9.

19 Ouchi N, Parker JL, Lugus J], Walsh K. Adipokines in inflammation and metabolic disease. Nat Rev Immunol 2011;11:85-97.

20 Richard C, Wadowski M, Goruk S et al. Individuals with obesity and type 2 diabetes have additional immune dysfunction compared with obese individuals who are metabolically healthy. BMJ Open Diabetes Res Care 2017;5:e000379.

21 Bandaru P, Rajkumar H, Nappanveettil G. The impact of obesity on immune response to infection and vaccine: an insight into plausible mechanisms. Endocrinol Metab Synd 2013;2:2. 
22 Ahn SY, Sohn SH, Lee SY et al. The effect of lipopolysaccharide-induced obesity and its chronic inflammation on influenza virus-related pathology. Environ Toxicol Pharmacol 2015;40:924-30.

23 Zhang HH, Halbleib M, Ahmad F, Manganiello VC, Greenberg AS. Tumor necrosis factor-alpha stimulates lipolysis in differentiated human adipocytes through activation of extracellular signal-related kinase and elevation of intracellular cAMP. Diabetes 2002;51:2929-35.

24 Bézaire V, Mairal A, Anesia R, Lefort C, Langin D. Chronic TNFalpha and cAMP pre-treatment of human adipocytes alter HSL, ATGL and perilipin to regulate basal and stimulated lipolysis. FEBS Lett 2009;583:3045-9.

25 Guilherme A, Virbasius JV, Puri V, Czech MP. Adipocyte dysfunctions linking obesity to insulin resistance and type 2 diabetes. Nat Rev Mol Cell Biol 2008;9:367-77.

26 Nieman DC, Henson DA, Nehlsen-Cannarella SL et al. Influence of obesity on immune function. J Am Diet Assoc 1999;99:294-9.

27 Nieman DC, Nehlsen-Cannarella SI, Henson DA et al. Immune response to obesity and moderate weight loss. Int J Obes Relat Metab Disord 1996;20:353-60.

28 O'Rourke RW, Kay T, Scholz MH et al. Alterations in T-cell subset frequency in peripheral blood in obesity. Obes Surg 2005;15:1463-68.

29 Tanaka S, Isoda F, Ishihara Y, Kimura M, Yamakawa T. T lymphopaenia in relation to body mass index and TNF-alpha in human obesity: adequate weight reduction can be corrective. Clin Endocrinol (Oxf) 2001;54:347-54.

30 Zimorovat A, Mohammadi M, Ramezani-Jolfaie N and SalehiAbargouei A. The healthy Nordic diet for blood glucose control: a systematic review and meta-analysis of randomized controlled clinical trials. Acta Diabetologica 2020;57:1-12.

31 Karlsson EA, Sheridan PA, Beck MA. Diet-induced obesity in mice reduces the maintenance of influenza-specific CD8 + memory $T$ cells. J Nutr 2010;140:1691-7.

32 Zhang AJ, To KK, Li C et al. Leptin mediates the pathogenesisi of severe 2009 pandemic influenza A (H1N1) infection associated with cytokine dysregulation in mice with diet-induced obesity. J Infect Dis 2013;207:1270-80.

33 Yang R, Barouch LA. Leptin signaling and obesity: cardiovascular consequences. Circ Res 2007;101:545-59.

34 Bennett BD, Solar GP, Yuan JQ et al. A role for leptin and its cognate receptor in hematopoiesis. Curr Biol 1996;6:1170-80.

35 Howard JK, Lord GM, Matarese G et al. Leptin protects mice from starvation-induced lymphoid atrophy and increases thymic cellularity in ob/ob mice. J Clin Invest 1999;104:1051-9.

36 Park S, Jeon J-H, Min B-K et al. Role of the pyruvate dehydrogenase complex in metabolic remodeling: differential pyruvate dehydrogenase complex functions in metabolism. Diabetes Metab ] 2018;42:270-81.

37 Maier H, Lopez R, Sanchez L et al. Obesity increases the duration of influenza a virus shedding in adults. J Infect Dis 2018;218:1378-82.
38 Klinkhammer J, Schnepf D, Ye L et al. INF-lambda prevents influenza virus spread from the upper airways to the lungs and limits virus transmission. elife 2018;7:e33354.

39 Honce R, Karlsson EA, Wohlgemuth $\mathrm{N}$ et al. Obesity-related microenvironment promotes emergence of virulent influenza virus strains. mBio 2020;11:1-16.

40 Yan J, Grantham M, Pantelic ] et al. Incetious virus in exhaled breath of syntomatic seasonal influenza cases from a college community. PNAS 2018;115:1081-6.

41 Dossett LA, Dageforde LA, Swenson BR et al. Obesity and sitespecific nosocomial infection risk in the intensive care unit. Surg Infect (Larchmt) 2009;10:137-42.

42 Jackson Leach R, Powis J, Baur LA et al. Clinical care for obesity: A preliminary survey of sixty-eight countries. Clin Obes 2020;10:e12357.

43 Maier HE, Lopez R, Sanchez $\mathrm{N}$ et al. Obesity increases the duration of influenza A virus shedding in adults. J Infect Dis 2018;218:1378-82.

44 Bhatraju PK, Ghassemieh B], Michelle Nichols M et al. Covid-19 in critically ill patients in the Seattle region-Case Series. N Engl J Med 2020;382:2012-22

45 Qingxian C, Fengjuan C, Fang L et al. Obesity and COVID-19 severity in a designated hospital in Shenzhen, China. SSRN 2020, preprint (doi: 10.2139/ssrn.3556658).

46 Peng YD, Meng K, Guan HQ et al. Clinical characteristics and outcomes of 112 cardiovascular disease patients infected by 2019nCoV. Zhonghua Xin Xue Guan Bing Za Zhi 2020;48:E004.

47 Movahed MR, Khoubyari R, Hashemzadeh M, Hashemzadeh M. Obesity is strongly and independently associated with a higher prevalence of pulmonary embolism. Respir Investig 2019;57:376-9.

48 Docherty A, Harrison E, Green C et al. Features of 16,749 hospitalised UK patients with COVID-19 using the ISARIC WHO Clinical Characterisation Protocol. medRxiv 2020, 2020.04.23.20076042.

49 Williamson E, Walker AJ, Bhaskaran KJ et al. OpenSAFELY: factors associated with COVID-19-related hospital death in the linked electronic health records of 17 million adult NHS patients. medRxiv 2020 2020.05.06.20092999.

50 Petrilli CM, Jones SA, Yang J et al. Factors associated with hospitalization and critical illness among 4,103 patients with COVID-19 disease in New York City. medRxiv 2020, 2020.04.08.20057794.

Address for correspondence: Dr Ahmed Albashir, Faculty of Medicine, University of Gezira, Wad Medani City, Gezira State, Sudan.

Email: drahmedabdalazim1992@yahoo.com 